\title{
BIRTH-DEATH CHAINS AND THE LOCAL TIME OF BROWNIAN MOTION
}

\section{GREG MARKOWSKY}

(Received 28 July 2011)

\section{Abstract}

A connection between Brownian motion and birth-death chains is explored. Several results concerning birth-death chains are shown to be consequences of well-known results on Brownian motion.

2010 Mathematics subject classification: primary 60J10; secondary 60J65.

Keywords and phrases: birth-death chain, Markov chain, Brownian motion, local time.

\section{Introduction}

Let $X_{m}$ be a Markov chain taking values on the nonnegative integers with the following transition probabilities for $n \neq 0$ :

$$
p_{n j}= \begin{cases}r_{n} & \text { if } j=n+1, \\ l_{n} & \text { if } j=n-1, \\ 0 & \text { if }|n-j| \neq 1,\end{cases}
$$

with $l_{n}+r_{n}=1$. We refer to such a Markov chain as a birth-death chain. (One may also find the term state-dependent random walk in the literature.) We set $p_{00}=1$ and $p_{0 j}=0$ for any $j \neq 0$. Let $k$ be the starting point of the birth-death chain, so $X_{0}=k$ almost surely. Let $\Delta$ denote the extinction time of $X_{m}$. That is, $\Delta=\inf _{m>0}\left\{X_{m}=0\right\}$, with $\Delta=\infty$ if $X_{m}$ never reaches 0 . In [5], a technique was given for realizing $X_{m}$ as a Brownian motion stopped at a certain sequence $\tau(m)$ of stopping times. This idea was used to prove the following two results, the first of which is well known.

Theorem 1.1. Let $P_{k}=P(\Delta<\infty)$, the extinction probability of $X_{m}$. Then

$$
P_{k}=\frac{\sum_{j=k}^{\infty} t_{j}}{\sum_{j=0}^{\infty} t_{j}}
$$

where this quotient is interpreted as being equal to 1 if the sums diverge.

The author is partially supported by Australian Research Council Grant DP0988483.

(C) 2011 Australian Mathematical Publishing Association Inc. 0004-9727/2011 \$16.00 
Theorem 1.2. If $t_{\infty}:=\lim _{n \rightarrow \infty}\left(\left(l_{1} \cdots l_{n}\right) /\left(r_{1} \cdots r_{n}\right)\right)$ exists, then $\lim _{m \rightarrow \infty} E\left[X_{m}\right]$ exists, and

$$
\lim _{m \rightarrow \infty} E\left[X_{m}\right]=\frac{1+\frac{l_{1}}{r_{1}}+\cdots+\frac{l_{1} \cdots l_{k-1}}{r_{1} \cdots r_{k-1}}}{t_{\infty}} .
$$

This result remains valid when $t_{\infty}=0$ or $\infty$, with the quotient being interpreted as equal to 0 when $t_{\infty}=\infty$ and equal to $\infty$ when $t_{\infty}=0$.

The purpose of this paper is to continue with this method by using known properties of Brownian motion to deduce several results concerning birth-death chains. The results presented can all be proved by more standard methods, but they will be seen to flow naturally from standard properties of Brownian motion, most notably the Tanaka and Itō-Tanaka formulas. In addition, the proofs given are quite short, although some time must be spent discussing the setup and relevant properties of Brownian motion. Let us begin by briefly recounting the construction given in [5]. Set

$$
t_{n}:=\frac{l_{1} l_{2} \cdots l_{n}}{r_{1} r_{2} \cdots r_{n}}
$$

and $x_{n}=\sum_{j=0}^{n-1} t_{j}$, where for $n=0$ these definitions are interpreted as $t_{0}=1, x_{0}=0$. Since the sequence $\left\{x_{n}\right\}_{n=0}^{\infty}$ is increasing it converges to a limit $x_{\infty}$, possibly infinite, as $n \rightarrow \infty$. Let $B_{t}$ be a Brownian motion stopped at the first time $\tau(\Delta)$ it hits 0 or $x_{\infty}$. Unless otherwise noted, the initial starting point of $B_{t}$ will be taken to be the point $x_{k} \in \mathcal{A}$, although we will use the standard notation $E_{z}$ to denote expectation conditioned on $B_{0}=z$. The recurrence properties of Brownian motion imply that $\tau(\Delta)<\infty$ almost surely. Set $\mathcal{A}=\bigcup_{n=0}^{\infty}\left\{x_{n}\right\}$. We define the stopping times $\tau(m)$ recursively by setting $\tau(0)=0$, and having defined $\tau(m)$ we let

$$
\tau(m+1)=\inf _{t>\tau(m)}\left\{B_{t} \in \mathcal{A}, \mathcal{B}_{\sqcup} \neq \mathcal{B}_{\tau(\mathfrak{l})}\right\} .
$$

That is, the $\tau(m) s$ are the the successive hitting times of points in $\mathcal{A}$. We see that the variables $B_{\tau(0)}, B_{\tau(1)}, B_{\tau(2)}, \ldots$ form a random process taking values in $\mathcal{A}$. Let $\phi: \mathcal{A} \rightarrow \mathbb{R}$ be defined by $\phi\left(x_{n}\right)=n$. It is shown in [5] that the strong Markov property of Brownian motion and the formula for the exit distribution of Brownian motion from an interval imply that $\phi\left(B_{\tau(0)}\right), \phi\left(B_{\tau(1)}\right), \phi\left(B_{\tau(2)}\right), \ldots$ is a realization of our birth-death chain. We therefore take $X_{m}=\phi\left(B_{\tau(m)}\right)$ in what follows. An important quantity for us will be the local time of Brownian motion, which is the density of the occupation measure of Brownian motion with respect to Lebesgue measure. That is, the local time $L_{t}^{x}$ satisfies

$$
L_{t}^{x} d x=\int_{0}^{t} 1_{B_{s} \in d x} d s .
$$

It is well known that $L_{t}^{x}$ exists and that

$$
L_{t}^{x}=\lim _{\varepsilon \rightarrow 0} \frac{1}{2 \varepsilon} \int_{0}^{t} 1_{\left|B_{s}-x\right|<\varepsilon} d s
$$


almost surely. The local time provides a measure of the amount of time that Brownian motion spends at a point. We will see that it can also be used to shed light upon the number of visits that a birth-death chain makes to a point, as well as the long-term behaviour of the chain.

The outline of the paper is as follows. We will begin by showing how Brownian local time can be used to give a quick derivation of the expected exit time of the birth-death chain from an interval. We will then prove an extension of Theorem 1.2 which relates to large stopping times. Some examples will be given of birth-death chains to which this result applies. We will conclude by showing that the Doob decomposition of the chain follows easily from the Itō-Tanaka formula for Brownian motion.

\section{The expected exit time from an interval}

For $0 \leq a<k<b$ let $\Gamma(a, b)=\inf _{m>0}\left\{X_{m}=a\right.$ or $\left.b\right\}$. We will calculate $E[\Gamma(a, b)]$. There is no loss of generality in assuming that the lower limit of our interval is 0 . The following result has probably been noted before, although this proof is likely to be new.

Theorem 2.1. Suppose that $0<k<M$. Then

$$
E[\Gamma(0, M)]=\frac{1}{x_{M}} \sum_{n=1}^{M-1} \frac{x_{M} \min \left(x_{k}, x_{n}\right)-x_{n} x_{k}}{r_{n} t_{n}} .
$$

REMARK 2.2. If $r_{n}=l_{n}=1 / 2$ for all $n, X_{m}$ is simple random walk, and (2.1) is readily seen to reduce to $k(M-k)$. This agrees with the formula obtained by applying the optional stopping theorem to the martingale $X_{m}^{2}-m$.

Proof. Set $G_{m}^{n}$ to be the number of times that $X$ is equal to $n$ on or before time $m-1$. It is clear that

$$
E[\Gamma(0, M)]=\sum_{n=1}^{M-1} E\left[G_{\Gamma(0, M)}^{n}\right] .
$$

We will use Brownian local time in order to determine $E\left[G_{\Gamma(0, M)}^{n}\right]$. The following lemma is the key calculation.

Lemma 2.3. Suppose that $a<y, z<b$. Let $\gamma(a, b)=\inf _{t>0}\left\{B_{t}=a\right.$ or $\left.b\right\}$. Then

$$
E_{z}\left[L_{\gamma(a, b)}^{y}\right]=\frac{2(b-a) \min (y-a, z-a)-2(y-a)(z-a)}{b-a} .
$$

Proof. As the right-hand side of (2.2) is unchanged when $\{a, y, z, b\}$ is replaced by $\{0, y-a, z-a, b-a\}$, it is enough to assume that $a=0$. Tanaka's formula (see [3] or [4]) states that

$$
\left|B_{t}-y\right|=\left|B_{0}-y\right|+\int_{0}^{t} \operatorname{sgn}\left(B_{s}-y\right) d B_{s}+L_{t}^{y} .
$$


The stochastic integral here is a martingale, and the optional stopping theorem can be applied. We find that

$$
E_{z}\left[L_{\gamma(0, b)}^{y}\right]=E_{z}\left|B_{\gamma(0, b)}-y\right|-|y-z| .
$$

Since $P\left(B_{\gamma(0, b)}=0\right)=(b-z) / b$ and $P\left(B_{\gamma(0, b)}=b\right)=z / b$, we see that $E_{z}\left|B_{\gamma(0, b)}-y\right|=$ $((b-z) / b) y+(z / b)(b-y)$. Thus

$$
\begin{aligned}
E_{z}\left[L_{\gamma(0, b)}^{y}\right] & =\frac{(b-z) y+z(b-y)-b|y-z|}{b} \\
& =\frac{b(y+z)-b|y-z|-2 y z}{b}=\frac{2 b \min (y, z)-2 y z}{b} .
\end{aligned}
$$

This proves the lemma.

We apply this lemma to our Brownian motion starting at $x_{k}$ in order to calculate $E\left[G_{\Gamma(0, M)}^{n}\right]$. The total expected local time accumulated at $x_{n}$ before hitting 0 or $x_{M}$ is given by $E_{x_{k}}\left[L_{\gamma\left(0, x_{M}\right)}^{x_{n}}\right]$. However, if we condition on $B_{\tau(m)}=x_{n}$ we see that the expected local time accumulated at $x_{n}$ between times $\tau(m)$ and $\tau(m+1)$ is $E_{x_{n}}\left[L_{\gamma\left(x_{n-1}, x_{n+1}\right)}^{x_{n}}\right]$. We therefore obtain

$$
E\left[G_{\Gamma(0, M)}^{n}\right]=\frac{E_{x_{k}}\left[L_{\gamma\left(0, x_{M}\right)}^{x_{n}}\right]}{E_{x_{n}}\left[L_{\gamma\left(x_{n-1}, x_{n+1}\right)}^{x_{n}}\right]} .
$$

We need therefore only apply Lemma 2.3 and simplify. We have

$$
\begin{aligned}
E_{x_{n}}\left[L_{\gamma\left(x_{n-1}, x_{n+1}\right)}^{x_{n}}\right] & =\frac{2\left(x_{n+1}-x_{n-1}\right)\left(x_{n}-x_{n-1}\right)-2\left(x_{n}-x_{n-1}\right)^{2}}{x_{n+1}-x_{n-1}} \\
& =\frac{2\left(x_{n+1}-x_{n}\right)\left(x_{n}-x_{n-1}\right)}{x_{n+1}-x_{n-1}} \\
& =\frac{2 t_{n} t_{n-1}}{t_{n}+t_{n-1}}=\frac{2 t_{n} t_{n-1}}{t_{n-1}\left(1+\frac{l_{n}}{r_{n}}\right)}=2 t_{n} r_{n},
\end{aligned}
$$

where we have used $r_{n}+l_{n}=1$. Plugging this into (2.3) and using Lemma 2.3 to evaluate $E_{x_{k}}\left[L_{\gamma\left(0, x_{M}\right)}^{x_{n}}\right]$ gives

$$
E\left[G_{\Gamma(0, M)}^{n}\right]=\frac{x_{M} \min \left(x_{n}, x_{k}\right)-x_{n} x_{k}}{x_{M} r_{n} t_{n}} .
$$

Summing over all $n$ between 1 and $M-1$ gives (2.1), and proves Theorem 2.1.

\section{An optional stopping-like theorem for large stopping times of birth-death chains}

We will prove the following result.

Theorem 3.1. Suppose that $t_{\infty}:=\lim _{n \rightarrow \infty}\left(\left(l_{1} \cdots l_{n}\right) /\left(r_{1} \cdots r_{n}\right)\right)$ exists. Let $\left\{T_{m}\right\}_{m=1}^{\infty}$ be a nondecreasing sequence of stopping times satisfying $E\left[T_{m}\right]<\infty$ such that $T_{m} \rightarrow \Delta$ 
almost surely. Then $\lim _{m \rightarrow \infty} E\left[X_{T_{m}}\right]$ exists, and

$$
\lim _{m \rightarrow \infty} E\left[X_{T_{m}}\right]=\frac{1+\frac{l_{1}}{r_{1}}+\cdots+\frac{l_{1} \cdots l_{k-1}}{r_{1} \cdots r_{k-1}}}{t_{\infty}} .
$$

Remark 3.2. This reduces to Theorem 1.2 when we take $T_{m}=m \wedge \Delta$. As before, the result is valid when $t_{\infty}=0$ or $\infty$, with the same interpretation as in Theorem 1.2.

Proof. We will assume that $t_{\infty} \in(0, \infty)$; easy adjustments can be made to the proof in order to handle the cases $t_{\infty}=0$ or $\infty$. Extend $\phi$ to a map from $\left[0, x_{\infty}\right)$ onto $[0, \infty)$ by requiring it to be continuous and linear on each interval $\left(x_{n}, x_{n+1}\right)$. Let $\phi_{n}^{\prime}$ be the derivative of $\phi$ on $\left(x_{n-1}, x_{n}\right)$. It was shown in [5] to be a consequence of the Itō-Tanaka formula (see [6, Theorem VI.1.5]) that

$$
X_{m}=\phi\left(B_{\tau(m)}\right)=k+\int_{0}^{\tau(m)} \phi^{\prime}\left(B_{s}\right) d B_{s}+\sum_{n=1}^{\infty} \frac{\phi_{n+1}^{\prime}-\phi_{n}^{\prime}}{2} L_{\tau(m)}^{x_{n}} .
$$

Let $T$ be a stopping time for $X_{m}$ with $E[T]<\infty$, and set $M(t)=\int_{0}^{t} \phi^{\prime}\left(B_{s}\right) d B_{s}$. We need to show that $E\left[M_{\tau(T)}\right]=0$. This is not quite trivial, since even if $T$ is bounded $\tau(T)$ will be unbounded. We must have control over $\tau(T)$ in order to apply the optional stopping theorem. The quadratic variation process of $M$, denoted $\langle M\rangle(t)$, is given by $\int_{0}^{t} \phi^{\prime}\left(B_{s}\right)^{2} d s$. We then have

$$
\begin{aligned}
E\left[\langle M\rangle_{\tau(m+1)}-\langle M\rangle_{\tau(m)} \mid B_{\tau(m)}=x_{n}\right] & \\
= & E\left[\int_{\tau(m)}^{\tau(m+1)} \phi^{\prime}\left(B_{s}\right)^{2} d s \mid B_{\tau(m)}=x_{n}\right] \\
= & \int_{x_{n-1}}^{x_{n+1}} \phi^{\prime}(x)^{2} E_{x_{n}}\left[L_{\gamma\left(x_{n-1}, x_{n+1}\right)}^{y}\right] d y \\
= & \frac{1}{t_{n-1}^{2}} \int_{x_{n-1}}^{x_{n}} E_{x_{n}}\left[L_{\gamma\left(x_{n-1}, x_{n+1}\right)}^{y}\right] d y+\frac{1}{t_{n}^{2}} \int_{x_{n}}^{x_{n+1}} E_{x_{n}}\left[L_{\gamma\left(x_{n-1}, x_{n+1}\right)}^{y}\right] d y \\
= & \frac{1}{t_{n-1}^{2}} \int_{x_{n-1}}^{x_{n}} \frac{2\left(x_{n+1}-x_{n-1}\right)\left(y-x_{n-1}\right)-2\left(x_{n}-x_{n-1}\right)\left(y-x_{n-1}\right)}{x_{n+1}-x_{n-1}} d y \\
& +\frac{1}{t_{n}^{2}} \int_{x_{n}}^{x_{n+1}} \frac{2\left(x_{n+1}-x_{n-1}\right)\left(x_{n}-x_{n-1}\right)-2\left(x_{n}-x_{n-1}\right)\left(y-x_{n-1}\right)}{x_{n+1}-x_{n-1}} d y \\
= & \frac{1}{t_{n-1}^{2}} \int_{x_{n-1}}^{x_{n}} \frac{2 t_{n}\left(y-x_{n-1}\right)}{t_{n}+t_{n-1}} d y+\frac{1}{t_{n}^{2}} \int_{x_{n}}^{x_{n+1}} \frac{2 t_{n-1}\left(x_{n+1}-y\right)}{t_{n}+t_{n-1}} d y \\
= & \frac{1}{t_{n-1}^{2}}\left(\frac{t_{n} t_{n-1}^{2}}{t_{n}+t_{n-1}}\right)+\frac{1}{t_{n}^{2}}\left(\frac{t_{n}^{2} t_{n-1}}{t_{n}+t_{n-1}}\right)=1 .
\end{aligned}
$$

The second equality is due to the occupation times formula [3, Theorem 8.10], and the fourth equality is Lemma 2.3. It follows easily from this that $E\left[\langle M\rangle_{\tau(T)}\right]=E[T]$. 
This tells us that the stopped process $M(t \wedge \tau(T))$ is uniformly integrable (see, for example [3, Corollary 7.8]), and thus that $E\left[M_{\tau(T)}\right]=E\left[M_{0}\right]=0$ by [3, Theorem 7.15]. We obtain

$$
E\left[X_{T_{m}}\right]=k+\sum_{n=1}^{\infty} \frac{\phi_{n+1}^{\prime}-\phi_{n}^{\prime}}{2} E_{x_{k}}\left[L_{\tau\left(T_{m}\right)}^{x_{n}}\right] .
$$

As $m \rightarrow \infty, T_{m} \nearrow \Delta$ and $E\left[L_{\tau\left(T_{m}\right)}^{x_{n}}\right] \nearrow E\left[L_{\tau(\Delta)}^{x_{n}}\right]$. Letting $b \rightarrow \infty$ in Lemma 2.3 gives $E_{x_{k}}\left[L_{\tau(\Delta)}^{x_{n}}\right]=2 \min \left(x_{n}, x_{k}\right)$. If we assume that $\sum_{n=1}^{\infty}\left|\phi_{n+1}^{\prime}-\phi_{n}^{\prime}\right|<\infty$, then we can apply the dominated convergence theorem (to the functions $\left(\left(\phi_{n+1}^{\prime}-\phi_{n}^{\prime}\right) / 2\right) E_{x_{k}}\left[L_{\tau\left(T_{m}\right)}^{x_{n}}\right]$ defined on $\mathbb{N}$ ) to conclude that

$$
\begin{aligned}
\lim _{m \rightarrow \infty} E\left[X_{T_{m}}\right] & =k+\sum_{n=1}^{\infty} \frac{\phi_{n+1}^{\prime}-\phi_{n}^{\prime}}{2} E_{x_{k}}\left[L_{\tau(\Delta)}^{x_{n}}\right] \\
& =k+\sum_{n=1}^{k-1}\left(\phi_{n+1}^{\prime}-\phi_{n}^{\prime}\right) x_{n}+x_{k} \sum_{n=k}^{\infty}\left(\phi_{n+1}^{\prime}-\phi_{n}^{\prime}\right) \\
& =k+\phi_{k}^{\prime} x_{k}-\phi_{1}^{\prime} x_{1}-\sum_{n=1}^{k-1} \phi_{n+1}^{\prime}\left(x_{n+1}-x_{n}\right)+x_{k}\left(\phi_{\infty}^{\prime}-\phi_{k}^{\prime}\right) .
\end{aligned}
$$

Using $x_{n+1}-x_{n}=t_{n}=1 / \phi_{n+1}$ and $\phi_{1}^{\prime} x_{1}=1$, this reduces to

$$
\lim _{m \rightarrow \infty} E\left[X_{T_{m}}\right]=x_{k} \phi_{\infty}^{\prime} .
$$

Recalling the values for $x_{k}$ and $\phi_{\infty}^{\prime}$, this is seen to be equivalent to (3.1). It remains to remove the restriction $\sum_{n=1}^{\infty}\left|\phi_{n+1}^{\prime}-\phi_{n}^{\prime}\right|<\infty$. If there is a sequence of numbers $N_{m}$ such that $\sup _{0 \leq m^{\prime} \leq T_{m}} X_{m^{\prime}} \leq N_{m}$ almost surely, then we can use [5, Lemma 3] and follow steps (22) through (26) of [5] in order to obtain the desired convergence. Suppose now that $T_{m}$ is an arbitrary sequence of stopping times as in the statement of the theorem. As $E\left[T_{m}\right]<\infty$, there is a number $H_{m}$ such that if $S_{m}=T_{m} \wedge H_{m}$, then $E\left[T_{m}-S_{m}\right]<1 / m$. Since $\left|X_{m+1}-X_{m}\right|=1$ almost surely, we see that

$$
\left|E\left[X_{T_{m}}\right]-E\left[X_{S_{m}}\right]\right| \leq E\left[T_{m}-S_{m}\right]<\frac{1}{m} .
$$

Since $\sup _{0 \leq m^{\prime} \leq S_{m}} X_{m^{\prime}} \leq k+H_{m}$, we see by our previous work that $\lim _{m \rightarrow \infty} E\left[X_{S_{m}}\right]=$ $x_{k} / t_{\infty}$, and it follows that the same is true for $\lim _{m \rightarrow \infty} E\left[X_{T_{m}}\right]$. This completes the proof of the theorem.

\section{Examples to which Theorem 3.1 can be applied}

Example 4.1. This example appeared in [5]. Let $l_{n}=n /(2 n+1), r_{n}=(n+1) /(2 n+1)$ for $n \geq 1$. Then $t_{n}=1 /(n+1)$, so that $t_{\infty}=0$. On the other hand, $x_{\infty}=1+\sum_{n=1}^{\infty} t_{n}=\infty$. We see that the birth-death chain $X_{m}$ built upon these transition probabilities has an extinction probability of 1 , but $E\left[X_{m}\right] \rightarrow \infty$ as $m \rightarrow \infty$.

Example 4.2. Suppose that there is an $M \geq 1$ such that $t_{n}=r_{n}=1 / 2$ for all $n>M$. Then $t_{\infty}=\left(l_{1} \cdots l_{M}\right) /\left(r_{1} \cdots r_{M}\right)$, and so, for a sequence $\left\{T_{m}\right\}_{m=1}^{\infty}$ satisfying the 
requirements for our theorem,

$$
E\left[X_{T_{m}}\right] \rightarrow \frac{1+\frac{l_{1}}{r_{1}}+\cdots+\frac{l_{1} \cdots l_{k-1}}{r_{1} \cdots r_{k-1}}}{\frac{l_{1} \cdots l_{M}}{r_{1} \cdots r_{M}}} .
$$

ExAmple 4.3. In order for $t_{\infty}$ to exist and lie in $(0, \infty)$, it is clearly necessary that $r_{n}, l_{n} \rightarrow 1 / 2$ as $n \rightarrow \infty$, but it is not sufficient. Example 4.1 shows this, as $t_{\infty}=0$. Exchanging $r_{n}$ and $l_{n}$ in Example 4.1 will give the case where $t_{\infty}=\infty$. It is not hard to interlace these series in a way so that $t_{\infty}$ does not exist. The theory of infinite products (most complex analysis texts contain this; see, for example, [1]) gives us the standard necessary condition for $t_{\infty}$ to exist and lie in $(0, \infty)$. The following proposition is obtained.

Proposition 4.4. Suppose that

$$
\sum_{n=1}^{\infty}\left|1-\frac{l_{n}}{r_{n}}\right|<\infty
$$

Then $\lim _{m \rightarrow \infty} E\left[X_{T_{m}}\right]$ exists and lies in $(0, \infty)$, where $\left\{T_{m}\right\}_{m=1}^{\infty}$ is any nondecreasing sequence of stopping times satisfying $E\left[T_{m}\right]<\infty$ such that $T_{m} \rightarrow \Delta$ almost surely.

\section{The Doob decomposition of $X_{m}$}

Let $\mathcal{F}_{t}$ be the filtration for $B_{t}$. Equation (3.2) shows that the $\sigma$-fields $\mathcal{F}_{\tau_{m}}$ can be taken as the filtration for the birth-death chain $X_{m}$, although of course this is far from the smallest possible filtration as it contains a great deal of extra information concerning the behaviour of $B_{t}$. Equation (3.2) comes from the Doob-Meyer decomposition of $\phi\left(B_{t}\right)$, but is not the discrete-time Doob decomposition of $X_{m}$ (see [2]), since $L_{\tau(m)}^{x_{n}}$ is not $\mathcal{F}_{\tau(m-1)}$ measurable. On the other hand, it leads very easily to the Doob decomposition.

Theorem 5.1. The Doob decomposition of $X_{m}$ is given by

$$
X_{m}=\mathcal{M}_{m}+\sum_{n=1}^{\infty} G_{m}^{n}\left(r_{n}-l_{n}\right)
$$

where $\mathcal{M}_{m}$ is the martingale given by

$$
\mathcal{M}_{m}=k+\int_{0}^{\tau(m)} \phi^{\prime}\left(B_{s}\right) d B_{s}+\sum_{n=1}^{\infty} \frac{\phi_{n+1}^{\prime}-\phi_{n}^{\prime}}{2}\left(L_{\tau(m)}^{x_{n}}-2 G_{m}^{n} r_{n} t_{n}\right) .
$$

Proof. By the standard arguments [2, Lemma 6.10] we have $X_{m}=M_{m}+A_{m}$, where $M_{m}$ is a martingale and $A_{m}=\sum_{m^{\prime}=1}^{m} E\left[X_{m}-X_{m^{\prime}-1} \mid \mathcal{F}_{\tau\left(m^{\prime}-1\right)}\right]$. Since $\int_{0}^{\tau(m)} \phi^{\prime}\left(B_{s}\right) d B_{s}$ is a martingale, (3.2) shows that

$$
E\left[X_{m}-X_{m^{\prime}-1} \mid \mathcal{F}_{\tau\left(m^{\prime}-1\right)}\right]=E\left[\sum_{n=1}^{\infty} \frac{\phi_{n+1}^{\prime}-\phi_{n}^{\prime}}{2}\left(L_{\tau\left(m^{\prime}\right)}^{x_{n}}-L_{\tau\left(m^{\prime}-1\right)}^{x_{n}}\right) \mid \mathcal{F}_{\tau\left(m^{\prime}-1\right)}\right]
$$


The result then follows from the following calculation:

$$
\begin{aligned}
& \frac{\phi_{n+1}^{\prime}-\phi_{n}^{\prime}}{2} \sum_{m^{\prime}=1}^{m} E\left[L_{\tau\left(m^{\prime}\right)}^{x_{n}}-L_{\tau\left(m^{\prime}-1\right)}^{x_{n}} \mid \mathcal{F}_{\tau\left(m^{\prime}-1\right)}\right] \\
& =\frac{\phi_{n+1}^{\prime}-\phi_{n}^{\prime}}{2} \sum_{m^{\prime}=1}^{m} E_{x_{n}}\left[L_{\gamma\left(x_{n-1}, x_{n+1}\right)}^{x_{n}}\right] 1_{\left\{B_{\tau\left(m^{\prime}-1\right)}=x_{n}\right\}} \\
& =\frac{\phi_{n+1}^{\prime}-\phi_{n}^{\prime}}{2} \sum_{m^{\prime}=1}^{m} 2 r_{n} t_{n} 1_{\left\{B_{\tau\left(m^{\prime}-1\right)}=x_{n}\right\}} \\
& =\frac{\phi_{n+1}^{\prime}-\phi_{n}^{\prime}}{2} 2 r_{n} t_{n} G_{m}^{n} \\
& =\left(\frac{1}{t_{n}}-\frac{1}{t_{n-1}}\right) r_{n} t_{n} G_{m}^{n}=\left(r_{n}-l_{n}\right) G_{m}^{n} .
\end{aligned}
$$

Note that the first equality above is due to the independent increments and strong Markov property of Brownian motion, while the second follows from Lemma 2.3 (see (2.4)).

\section{Acknowledgement}

I would like to thank Yoni Nazarathy for an invitation which contributed to the development in this paper.

\section{References}

[1] L. V. Ahlfors, Complex Analysis: An Introduction to the Theory of Analytic Functions of One Complex Variable (McGraw-Hill, New York, 1966).

[2] O. Kallenberg, Foundations of Modern Probability (Springer, New York, 2002).

[3] F. C. Klebaner, Introduction to Stochastic Calculus with Applications (Imperial College Press, London, 2005).

[4] M. B. Marcus and J. Rosen, Markov Processes, Gaussian Processes and Local Times (Cambridge University Press, Cambridge, 2006).

[5] G. Markowsky, 'Applying Brownian motion to the study of birth-death chains', Statist. Probab. Lett. 81(8) (2011), 1173-1178.

[6] D. Revuz and M. Yor, Continuous Martingales and Brownian Motion (Springer, Berlin, 1999).

GREG MARKOWSKY, Department of Mathematical Sciences,

Monash University, Victoria, 3800, Australia

e-mail: gmarkowsky@gmail.com 